\title{
Einstellungen gegenüber gesellschaftlichen Institutionen und der Demokratie
}

\author{
Nora Karnick, Julia Simonson \& Clemens Tesch-Römer
}

\section{Kernaussagen}

Die Mehrheit der Bevölkerung in Deutschland vertraut wichtigen gesellschaftlichen Institutionen, wobei es deutliche Unterschiede zwischen den Institutionen gibt. Besonders hoch ist das Vertrauen in Polizei (90,1 Prozent) und Justiz (78,5 Prozent). Deutlich niedriger ist das Vertrauen in Bundestag (59,6 Prozent), Bundesregierung (58,6 Prozent) und Europäisches Parlament (55,7 Prozent). Den politischen Parteien vertraut nur ein vergleichsweise geringer Anteil (36,4 Prozent).

Bei jüngeren Menschen und Personen mit hoher Bildung ist das Institutionenvertrauen höher als bei älteren Menschen und Menschen mit niedriger Bildung. In der Altersgruppe der 50- bis 64-Jährigen sind die Anteile von Personen, die der Bundesregierung, dem Bundestag, dem Europäischen Parlament oder den Parteien vertrauen, am geringsten. Ähnlich ist es bei Personen mit niedriger Bildung: Hier vertraut weniger als die Hälfte der Bevölkerung den genannten Institutionen. Unterschiede zwischen Frauen und Männern sind nur gering.

Bei freiwillig Engagierten ist der Anteil von Personen, die den Institutionen vertrauen, höher als bei Nicht-Engagierten. Mit einer Differenz von 8,8 Prozentpunkten ist der Unterschied beim Vertrauen in die Justiz am größten. Die geringsten Unterschiede zwischen Engagierten und Nicht-Engagierten sind mit einer Differenz von jeweils 3,5 Prozentpunkten beim Vertrauen in Polizei und Parteien zu finden. 
Eine positive Einstellung zur Demokratie als Regierungsform äußern neun von zehn Personen. Zufrieden mit dem Funktionieren der Demokratie in Deutschland sind etwas mehr als zwei Drittel der Menschen. Die Kombination beider Abfragen ergibt, dass der Großteil der Menschen die Demokratie als gute Regierungsform bewertet und gleichzeitig mit dem Funktionieren der Demokratie in Deutschland zufrieden ist $(66,4$ Prozent).

Bei jüngeren Personen ist eine positive Einstellung zur Demokratie anteilig häufiger als bei älteren Menschen. Personen ab 50 Jahren geben anteilig deutlich häufiger als Personen zwischen 14 und 49 Jahren an, die Demokratie für keine gute Regierungsform zu halten und mit dem Funktionieren der Demokratie nicht zufrieden zu sein.

Personen, die aktuell die Schule besuchen, bewerten die Demokratie anteilig am häufigsten als gute Regierungsform und sind mit dem Funktionieren in Deutschland zufrieden. Personen mit hoher Bildung und Personen mit mittlerer Bildung geben dies anteilig etwas seltener an. Bei Personen mit niedriger Bildung ist hier der geringste Anteil festzustellen.

Bei freiwillig Engagierten ist der Anteil der Personen, die die Demokratie als gute Regierungsform bewerten, höher als bei Nicht-Engagierten. Auch die Zufriedenheit mit der Demokratie ist bei freiwillig Engagierten höher als bei Nicht-Engagierten. Unter den Engagierten geben 71,0 Prozent an, die Demokratie als gute Regierungsform zu bewerten und mit dem Funktionieren der Demokratie in Deutschland zufrieden zu sein. Unter den Nicht-Engagierten beträgt dieser Anteil 63,3 Prozent. 


\subsection{Einleitung}

In der politischen und öffentlichen Diskussion wird vielfach davon ausgegangen, dass gesellschaftliche Teilhabe zu mehr Zusammenhalt in der Gesellschaft führe und präventiv gegen die Verbreitung extremistischer Einstellungen wirke (Bundesregierung 2016). Damit kann angenommen werden, dass freiwilliges Engagement ein wichtiges Element sozialer Teilhabe sowie Ausdruck einer starken Zivilgesellschaft und einer stabilen Demokratie ist (zum Beispiel Alscher et al. 2021; Putnam 2000; Simonson \& Vogel 2017). Empirische Befunde lassen auf einen Zusammenhang zwischen freiwilligem Engagement und einer positiven Einstellung zur Demokratie als Regierungsform schließen. So stellte der Soziologe und Politikwissenschaftler Robert Putnam fest, dass zwischen freiwilligem Engagement und dem Vertrauen in die Gesellschaft ein positiver Zusammenhang bestehe (Putnam 2000). Zudem kann die Beteiligung der Bevölkerung am gesellschaftlichen Geschehen zu einer höheren Akzeptanz politischer Entscheidungen führen (Simonson et al. 2017).

Die gesellschaftliche Teilhabe der Menschen durch freiwilliges Engagement und unterschiedliche Formen der politischen Partizipation ist aber nur dann eine Ressource für demokratische Prozesse, wenn sie auf demokratischen Prinzipien beruht und demokratische Ziele verfolgt (Geißel 2006; Putnam 2000). Das freiwillige Engagement von Personen kann in unterschiedlichen Kontexten und aus unterschiedlichen Motivationen heraus stattfinden. So kann freiwilliges Engagement auch durch die Unzufriedenheit mit dem Funktionieren der Demokratie oder durch ein geringes Vertrauen in diese sowie in staatliche Institutionen motiviert sein (Decker et al. 2019). Resultiert aus dieser Motivlage im Extremfall ein Engagement, das danach strebt, die Demokratie zu schwächen, zu beschädigen oder sogar abzuschaffen, so wird in diesem Fall die Erwartung, dass freiwilliges Engagement den gesellschaftlichen $\mathrm{Zu}$ sammenhalt stärkt und die Demokratie festigt, nicht erfüllt. Für eine funktionierende Demokratie ist also nicht grundsätzlich entscheidend, ob Engagement überhaupt stattfindet, sondern mit welchen Überzeugungen die Menschen aktiv werden (van Deth 2013). Demokratiefeindliches Engagement stärkt die Demokratie nicht, sondern ist in der Lage, sie zu schwächen.

Es wird vielfach diskutiert, ob sich die Demokratie in Deutschland derzeit in einer Krise befindet (Jesse 2020; Kalb 2020; Küpper \& Zick 2020). Dies zeigt sich an der Debatte um die Grundrechtseinschränkungen aufgrund der Corona-Pandemie, die durch die Regierung und das Parlament auf Grundlage des Infektionsschutzgesetzes beschlossen wurden (Spickschen et al. 2020). Diese Maßnahmen sind bei einigen Menschen auf Unverständnis und Abwehr gestoßen und könnten die Einstellung zur Demokratie sowie das Vertrauen in wichtige gesellschaftliche Institutionen beeinflussen. Die Daten zu den in diesem Kapitel berichteten Ergebnissen wurden allerdings im Jahr 2019, also vor der Corona-Pandemie, erhoben. Somit können die hier dargestellten Ergebnisse die Entwicklung im Jahr 2020 nicht widerspiegeln, sondern beziehen sich auf den Zeitraum bis einschließlich November 2019. 
Doch auch schon vor den Debatten über politische Maßnahmen aufgrund der Corona-Pandemie wurde über eine Krise der Demokratie diskutiert. Studien zu den Einstellungen zur Demokratie aus den Jahren vor 2020 beziehen sich insbesondere auf die politische Debatte um den Zuzug von Geflüchteten seit 2015 (Stroppe \& Mauk 2021; Decker et al. 2019; Zick \& Küpper 2016). Aus diesem Ereignis resultierte einerseits freiwilliges Engagement, das sich speziell an Geflüchtete sowie Asylsuchende richtet (siehe Kapitel 10), andererseits aber führte es zu der verstärkten Mobilisierung einer politischen Gegenbewegung, mit Gruppierungen wie beispielsweise der ,Identitären Bewegung' oder Pegida, die antidemokratische Tendenzen und eine personelle sowie inhaltliche Überschneidung mit rechtsextremen Kreisen aufweist (Vorländer et al. 2017; Dietrich 2019; Pfahl-Traughber 2019).

Die Befunde verschiedener Studien bestätigen nach wie vor eine sehr große Präferenz der Menschen für die Demokratie als Staatsform; das Funktionieren der Demokratie in Deutschland wird im Vergleich dazu seltener als zufriedenstellend bewertet (Bertelsmann Stiftung 2019; Decker et al. 2018; Decker et al. 2019). Beide Indikatoren hängen miteinander zusammen. Die Bewertung der Demokratie als Prinzip kann durch die Zufriedenheit mit der Leistung beeinflusst werden (Busch 2020). In Deutschland zeigt sich eine deutliche Diskrepanz zwischen der Bewertung der Demokratie als Ideal und der Zufriedenheit mit ihrer praktischen Umsetzung (Zick et al. 2016). Diese Erkenntnis wird dadurch unterstützt, dass das Vertrauen in bestimmte, für die Demokratie elementare Institutionen, zum Teil niedrig ist. Es ist davon auszugehen, dass die Zufriedenheit mit der Umsetzung der Demokratie eng mit dem Vertrauen in wichtige staatliche Institutionen verbunden ist.

Unterschiedliche Sozialisationsprozesse sowie die individuelle Lebenssituation, die eng an das Geschlecht, das Alter und die Bildung von Personen geknüpft sind, können einen wichtigen Faktor bei dem Vertrauen in Institutionen und der Einstellung gegenüber der Demokratie darstellen. So zeigen sich zwischen den verschiedenen Bevölkerungsgruppen zum Teil deutliche Unterschiede. Der Bildungsstand einer Person spielt eine wichtige Rolle und auch zwischen den Altersgruppen zeigen sich Unterschiede im Institutionenvertrauen. Zwischen Frauen und Männern zeigen sich hinsichtlich des Institutionenvertrauens dagegen nur geringe Unterschiede (Decker et al. 2019).

Im Deutschen Freiwilligensurvey werden 2019 erstmals sowohl das Vertrauen in Institutionen als auch Einstellungen zur Demokratie erhoben. Es wird das Vertrauen $\mathrm{zu}$ sechs zentralen Institutionen des gesellschaftlichen Lebens erfragt (Polizei, Justiz, Bundestag, Bundesregierung, Europäisches Parlament, politische Parteien). Bei den Einstellungen zur Demokratie geht es in einem ersten Schritt darum, ob die Befragten die Demokratie allgemein für eine gute Regierungsform halten, und in einem zweiten Schritt, wie das Funktionieren der Demokratie speziell in Deutschland bewertet wird. Es ist davon auszugehen, dass Personen, die die Demokratie grundsätzlich ablehnen, auch mit ihrem Funktionieren nicht zufrieden sind. Unter den Personen, die der Demokratie ablehnend gegenüberstehen, können aber durchaus Personen sein, 
die die Performanz der Demokratie für die eigene Lebenszufriedenheit als positiv bewerten und daher angeben mit dem Funktionieren zufrieden zu sein. Ebenso können Personen, die das Prinzip der Demokratie grundsätzlich unterstützen, mit dem aktuellen Funktionieren nicht zufrieden sein. Und es gibt diejenigen, die die Demokratie als gute Regierungsform bewerten und mit dem Funktionieren der Demokratie in Deutschland zufrieden sind. Beide Indikatoren werden daher sowohl einzeln als auch zusammen ausgewertet. Dies ermöglicht es, die Einstellungen zur Demokratie differenziert abzubilden und potenziell demokratiefeindliche Einstellungen in der Bevölkerung Deutschlands ab 14 Jahren zu identifizieren. Dabei wird eine Differenzierung nach Geschlecht, Alter und Bildung vorgenommen, um die individuellen Lebenssituationen der Personen genauer betrachten und statistisch signifikante Auffälligkeiten identifizieren zu können.

Darüber hinaus werden das Institutionenvertrauen und die Demokratieeinstellungen im Kontext des freiwilligen Engagements betrachtet. Es ist zwar nicht möglich, mit den Daten des Freiwilligensurveys 2019 die Frage zu beantworten, ob Engagierte mit ihrem freiwilligen Engagement demokratische oder antidemokratische Ziele verfolgen. Es ist jedoch möglich, den Anteil der Engagierten mit hohem und geringem Vertrauen in die Institutionen und die Demokratie zu ermitteln und so ein potenziell demokratiefeindliches, aber auch ein potenziell die Demokratie stärkendes Engagement zu quantifizieren. Zudem können so auch Unterschiede zwischen Engagierten und Nicht-Engagierten hinsichtlich ihres Institutionenvertrauens und ihrer Demokratieeinstellung benannt werden.

Es sollen folgende Fragen untersucht werden:

a) Wie hoch sind die Anteile der Personen, die 2019 unterschiedlichen Institutionen der Demokratie vertrauen? Welche Unterschiede zeigen sich zwischen Frauen und Männern, zwischen den Altersgruppen und zwischen den Bildungsgruppen? Wie unterscheiden sich die Anteile zwischen Engagierten und Nicht-Engagierten?

b) Wie hoch sind die jeweiligen Anteile der Personen mit unterschiedlichen Einstellungen zur Demokratie allgemein und zur Demokratie in Deutschland 2019? Wie hoch sind die Anteile der unterschiedlichen Einstellungen zur Demokratie, wenn beide Einstellungen kombiniert betrachtet werden? Wie unterscheiden sich die Anteile nach Geschlecht, nach Altersgruppen und nach Bildungsgruppen?

c) Wie hoch sind die jeweiligen Anteile der Einstellungen zur Demokratie allgemein und der Einstellungen zur Demokratie in Deutschland 2019 nach Engagementstatus? Wie hoch sind die Anteile der unterschiedlichen Einstellungen zur Demokratie nach Engagementstatus, wenn beide Einstellungen kombiniert betrachtet werden? 


\subsection{Daten und Indikatoren}

Die Ergebnisse des vorliegenden Kapitels beruhen auf den Daten des Deutschen Freiwilligensurveys (FWS) und werden gewichtet dargestellt. Die Gewichtung zielt darauf ab, die Repräsentativität zu verbessern, um verallgemeinerbare Aussagen treffen zu können. In den Veröffentlichungen zu den Erhebungen der Jahre 1999 bis 2014 wurden im Rahmen des Freiwilligensurveys bislang die Gewichtungsmerkmale Geschlecht, Alter, Bundesland sowie Gemeindegrößenklasse herangezogen. Diese Gewichtung wird nun um das Gewichtungsmerkmal der schulischen Bildung ergänzt. Personen aus unterschiedlichen Bildungsgruppen nehmen mit unterschiedlicher Wahrscheinlichkeit an Umfragen teil; daher sind verschiedene Bildungsgruppen in den Daten über- beziehungsweise unterrepräsentiert. Die Gewichtung inklusive schulischer Bildung wird in diesem Bericht auf die Daten aller fünf Erhebungswellen von 1999 bis 2019 angewendet. Die Engagementquoten fallen durch dieses Vorgehen für alle Erhebungswellen niedriger aus als bisher berichtet; es ändert sich somit das Niveau, der Trend bleibt jedoch im Wesentlichen bestehen. Die Gewichtung der Daten inklusive Bildung wirkt sich auch auf andere Ergebnisse aus.

Alle Ergebnisse werden auf statistische Signifikanz getestet (verwendet wird ein Signifikanzniveau von $\mathrm{p}<0,05)$. Die Prüfung auf statistische Signifikanz zeigt an, ob ein anhand der Daten des Freiwilligensurveys gefundener Unterschied mit großer Wahrscheinlichkeit auch in der Grundgesamtheit, also beispielsweise in der Wohnbevölkerung Deutschlands, vorhanden ist (statistisch signifikant) oder nur zufällig in den Daten vorliegt (statistisch nicht signifikant).

Die dargestellten Ergebnisse fußen auf der Befragung des Freiwilligensurveys 2019, die von März bis November 2019 erfolgte. Somit ist es mithilfe der Daten des Freiwilligensurveys 2019 nicht möglich, Aussagen über spätere Entwicklungen wie beispielsweise die Auswirkungen der Corona-Pandemie auf das freiwillige Engagement zu tätigen.

Vertrauen in Institutionen: Das Institutionenvertrauen wird im Freiwilligensurvey 2019 erstmals erhoben. Die Frage nach dem Vertrauen in sechs spezifische Institutionen der Demokratie wird allen Befragten gestellt:

Ich nenne Ihnen jetzt eine Reihe von öffentlichen Einrichtungen oder Personengruppen. Sagen Sie mir bitte, ob Sie diesen Einrichtungen oder Personengruppen jeweils voll und ganz vertrauen, eher vertrauen, eher nicht vertrauen oder ganz und gar nicht vertrauen.

(1) der Polizei

(2) dem Bundestag

(3) den politischen Parteien

(4) der Justiz

(5) der Bundesregierung

(6) dem Europäischen Parlament 
Antwortkategorien (jeweils): vertraue voll und ganz; vertraue eher; vertraue eher nicht; vertraue ganz und gar nicht

Diese vier Antwortkategorien werden in der Ergebnisdarstellung zu zwei Kategorien zusammengefasst: Die Antwortmöglichkeiten, vertraue voll und ganz' und, vertraue eher' werden zu der Kategorie ,vertraue', die Antwortmöglichkeiten ,vertraue eher nicht' und ,vertraue ganz und gar nicht` zu der Kategorie ,vertraue nicht' gebündelt.

Einstellungen zur Demokratie: Auch die Fragen nach den Einstellungen zur Demokratie werden im Freiwilligensurvey 2019 erstmals gestellt und sind an alle Befragte gerichtet. Im Freiwilligensurvey 2019 wird erfasst, welche Einstellung zur Demokratie die Befragten allgemein haben und wie zufrieden sie im Speziellen mit dem Funktionieren der Demokratie in Deutschland sind.

Die Einstellung zur Demokratie allgemein wird wie folgt erfragt:

Einmal abgesehen davon, wie gut die Demokratie in Deutschland funktioniert: Halten Sie die Demokratie ganz allgemein für eine gute Regierungsform oder für eine nicht so gute Regierungsform?

Antwortkategorien: gute Regierungsform; nicht so gute Regierungsform

Die Fragestellung nach der Einstellung zur Demokratie in Deutschland lautet:

Sind Sie mit der Art und Weise, wie die Demokratie in Deutschland funktioniert, alles in allem... sehr zufrieden; eher zufrieden; eher nicht zufrieden; gar nicht zufrieden?

Die vier Antwortkategorien zur Zufriedenheit werden in der Ergebnisdarstellung zusammengefasst zu zwei Kategorien. Die Antwortmöglichkeiten ,sehr zufrieden und ,eher zufrieden' werden zu der Kategorie ,zufrieden', die Antwortmöglichkeiten ,eher nicht zufrieden' und , gar nicht zufrieden' zu der Kategorie ,nicht zufrieden' gebündelt.

Die Ergebnisse beider Abfragen zu den Einstellungen gegenüber der Demokratie allgemein und zur Demokratie in Deutschland werden in einem ersten Schritt einzeln und nach verschiedenen Merkmalen differenziert betrachtet. In einem zweiten Schritt werden die Antworten beider Fragen miteinander kombiniert dargestellt, sodass die Summe aller Ausprägungen 100 Prozent ergibt. Die zwei Fragen werden zusammen ausgewertet, indem vier Gruppen gebildet werden:

- Personen, die die Demokratie allgemein als eine nicht so gute Regierungsform betrachten und mit der Demokratie in Deutschland nicht zufrieden sind.

- Personen, die die Demokratie allgemein als eine nicht so gute Regierungsform betrachten und mit der Demokratie in Deutschland zufrieden sind. 
- Personen, die die Demokratie allgemein als eine gute Regierungsform betrachten und mit der Demokratie in Deutschland nicht zufrieden sind.

- Personen, die die Demokratie allgemein als eine gute Regierungsform betrachten und mit der Demokratie in Deutschland zufrieden sind.

Die verwendeten Differenzierungsmerkmale werden in Kapitel 2 beschrieben.

\subsection{Das Vertrauen in unterschiedliche Institutionen der Demokratie}

Betrachten wir das Vertrauen in unterschiedliche gesellschaftliche Institutionen unter allen Befragten, zeigen sich sehr deutliche Unterschiede zwischen den Institutionen (Abbildung 14-1). Die Polizei genießt mit 90,1 Prozent das größte Vertrauen, gefolgt von der Justiz mit 78,5 Prozent und dem Bundestag mit 59,6 Prozent. Der Bundesregierung und dem Europäischen Parlament vertraut jeweils mehr als die Hälfte der Personen. Lediglich den Parteien vertraut mit 36,4 Prozent nur ein gutes Drittel.

Abbildung 14-1 Vertrauen in unterschiedliche Institutionen 2019, gesamt (Basis: alle Befragten)

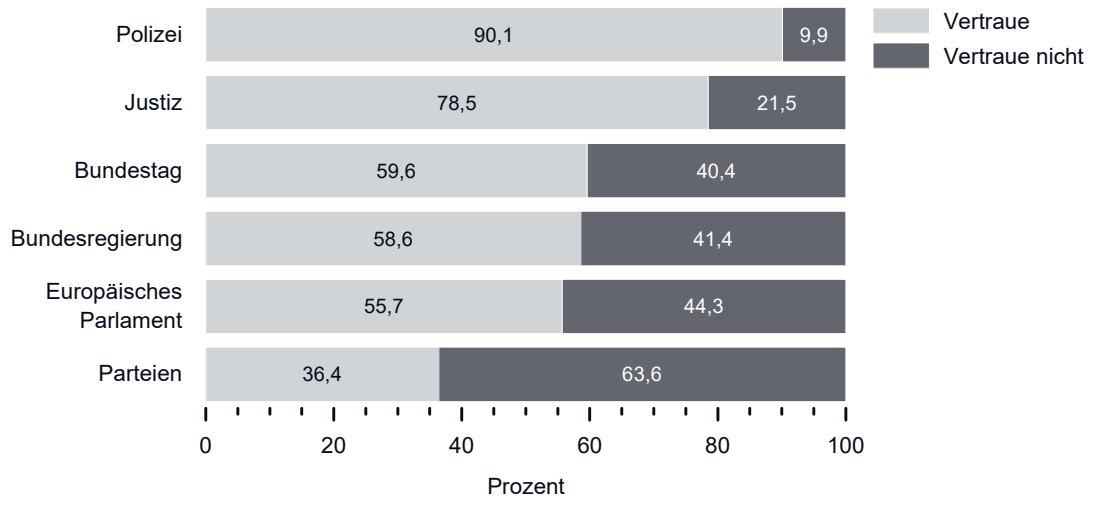

Quelle: FWS 2019, gewichtet, eigene Berechnungen (DZA). Basis: alle Befragten ( $n=26.404-27.414)$.

Zwischen Frauen und Männern zeigen sich nur geringe Unterschiede im Vertrauen in diese Institutionen (Abbildung 14-2). Mit Blick auf das Vertrauen in Justiz, Bundestag und Parteien finden sich keine statistisch signifikanten Geschlechterunterschiede. Den weiteren Institutionen vertrauen Frauen anteilig etwas häufiger als Männer: Unterschiede finden sich dahingehend beim Vertrauen in die Polizei, die Bundesregierung und das Europäische Parlament. 
Abbildung 14-2 Vertrauen in unterschiedliche Institutionen 2019, nach Geschlecht (Basis: alle Befragten)

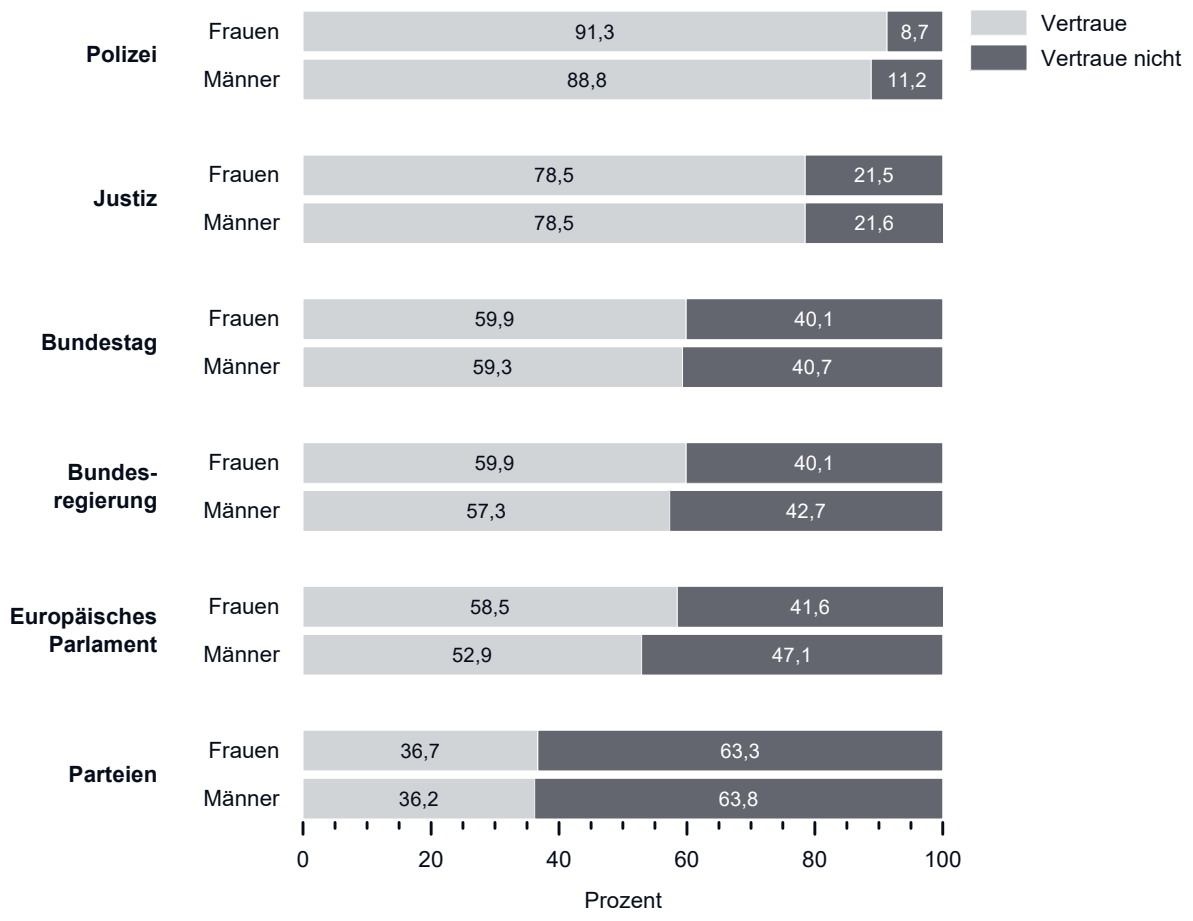

Quelle: FWS 2019, gewichtet, eigene Berechnungen (DZA). Basis: alle Befragten ( $n=26.404-27.414$ ).

Hinweis: Abweichungen in den Prozentwerten ergeben sich durch das Auf- und Abrunden der Werte.

Statistisch signifikanter Geschlechterunterschied bei dem Vertrauen in Polizei, Bundesregierung sowie Europäisches Parlament. Kein statistisch signifikanter Unterschied zwischen Frauen und Männern beim Vertrauen in Justiz, Bundestag sowie Parteien.

Hinsichtlich des Vertrauens in die Institutionen zeigen sich unterschiedliche Muster nach Altersgruppen (Abbildung 14-3). Das Vertrauen in die Polizei ist in allen Altersgruppen sehr groß, allerdings vertrauen die Personen zwischen 14 und 29 Jahren der Polizei mit 87,2 Prozent anteilig seltener als die Befragten aller anderen Altersgruppen, deren Anteile jeweils über 90 Prozent liegen. Der Justiz vertrauen die 14- bis 29-Jährigen mit 84,4 Prozent anteilig am häufigsten, gefolgt von den 30- bis 49-Jährigen und den 50- bis 64-Jährigen. Anteilig am seltensten vertrauen die Personen ab 65 Jahren der Justiz mit 71,1 Prozent. Bei den im engeren Sinne politischen Institutionen Bundestag, Bundesregierung, Europäisches Parlament und Parteien sind die Muster der Unterschiede zwischen den Altersgruppen - auf jeweils unterschiedlichem Niveau - sehr ähnlich. Diesen vier Institutionen vertrauen die 14- bis 29-Jährigen jeweils anteilig häufiger als die anderen Altersgruppen; die 50- bis 64-Jährigen vertrauen diesen Institutionen im Vergleich zu allen anderen Altersgruppen anteilig am seltensten. Die Altersgruppen der 30- bis 49-Jährigen sowie der 65-Jährigen 
Abbildung 14-3 Vertrauen in unterschiedliche Institutionen 2019, nach Alter (Basis: alle Befragten)

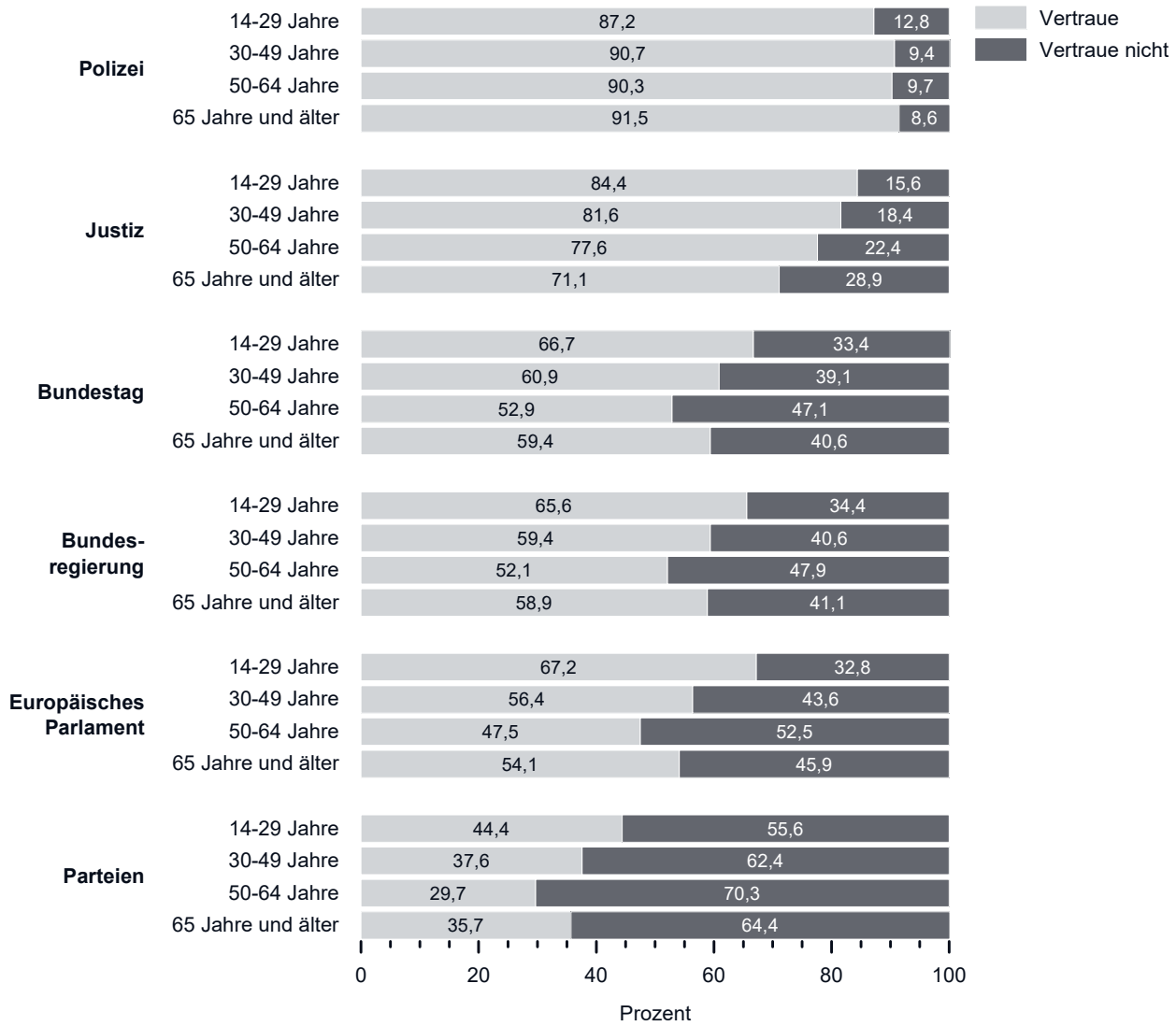

Quelle: FWS 2019, gewichtet, eigene Berechnungen (DZA). Basis: alle Befragten ( $n=26.404-27.414)$.

Hinweis: Abweichungen in den Prozentwerten ergeben sich durch das Auf- und Abrunden der Werte.

Polizei: Statistisch signifikanter Unterschied zwischen den 14- bis 29-Jährigen und allen anderen Altersgruppen. Kein statistisch signifikanter Unterschied zwischen den weiteren Altersgruppen.

Justiz: Statistischer signifikanter Unterschied zwischen allen Altersgruppen.

Bundestag/Bundesregierung/Europäisches Parlament/Parteien: Kein statistisch signifikanter Unterschied zwischen den 30- bis 49-Jährigen und den 65-Jährigen und Älteren. Alle weiteren Unterschiede sind statistisch nicht signifikant.

und Älteren unterscheiden sich hinsichtlich des Vertrauens in diese vier Institutionen nicht statistisch signifikant voneinander.

Das Vertrauen in Institutionen hängt sehr stark mit dem Bildungsstand zusammen (Abbildung 14-4). Bei den Personen mit hoher Bildung sowie denen, die aktuell noch die Schule besuchen, ist der Anteil derjenigen, die Vertrauen in die jeweiligen Institutionen haben, deutlich höher als bei Personen mit niedriger Bildung. Die Polizei genießt über alle Bildungsgruppen hinweg ein sehr hohes Vertrauen; die Unterschiede zwischen den Bildungsgruppen sind hierbei nur gering. Das Vertrauen 
in die Justiz und den Bundestag, differenziert nach Bildungsgruppen, weist ein gleiches Muster auf, jedoch auf einem unterschiedlichen Niveau. Der Justiz vertrauen die Menschen anteilig weitaus häufiger als dem Bundestag. Das Vertrauen in die Justiz und den Bundestag ist bei Personen mit niedriger Bildung am wenigsten verbreitet, gefolgt von den Personen mit mittlerer Bildung. Deutlich höher ist das Vertrauen in die Justiz und den Bundestag bei Personen, die aktuell eine Schule besuchen, sowie bei Personen mit hoher Bildung. Das Vertrauen in die Bundesregierung sowie

Abbildung 14-4 Vertrauen in unterschiedliche Institutionen 2019, nach Bildung (Basis: alle Befragten)

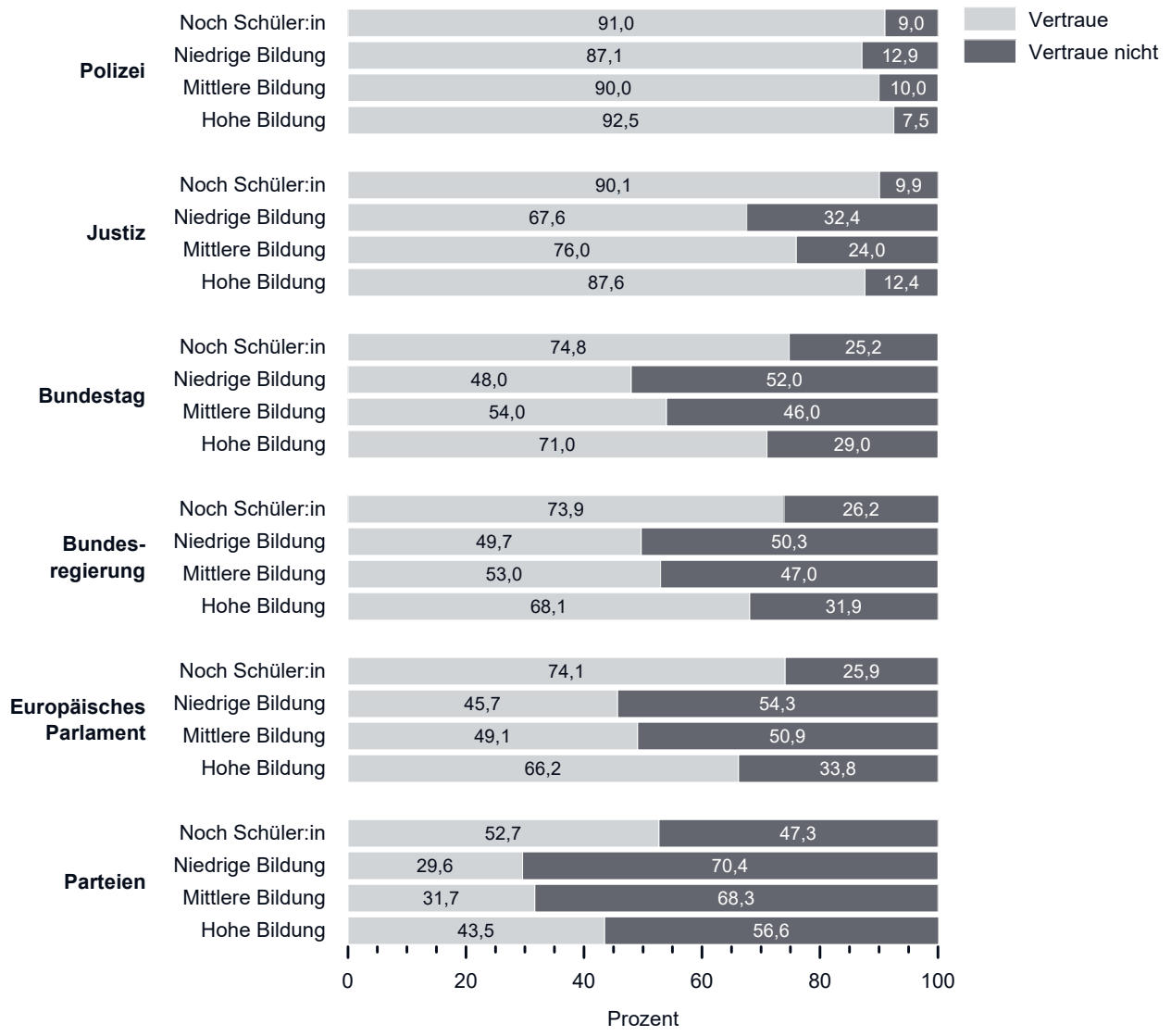

Quelle: FWS 2019, gewichtet, eigene Berechnungen (DZA). Basis: alle Befragten ( $n=26.400-27.409$ ).

Hinweis: Abweichungen in den Prozentwerten ergeben sich durch das Auf- und Abrunden der Werte.

Polizei: Statistisch signifikanter Unterschied zwischen Personen mit niedriger Bildung und allen anderen Bildungsgruppen. Statistisch signifikanter Unterschied zwischen Personen mit mittlerer und hoher Bildung.

Justiz/Bundestag: Statistisch signifikanter Unterschied zwischen Personen mit niedriger Bildung und allen anderen Bildungsgruppen. Statistisch signifikanter Unterschied zwischen Personen mit mittlerer Bildung und allen anderen Bildungsgruppen.

Bundesregierung/Europäisches Parlament: Statistisch signifikanter Unterschied zwischen allen Bildungsgruppen.

Parteien: Kein statistisch signifikanter Unterschied zwischen Personen mit niedriger und mittlerer Bildung; alle weiteren Unterschiede

zwischen den Bildungsgruppen sind statistisch signifikant. 
in das Europäische Parlament bewegt sich auf einem ähnlichen Niveau und weist das gleiche Muster auf. Der Bundesregierung und dem Europäischen Parlament vertrauen die Personen, die noch die Schule besuchen, anteilig am häufigsten, gefolgt von Personen mit hoher Bildung, Personen mit mittlerer und Personen mit niedriger Bildung. Das geringste Vertrauen haben die Befragten aller Bildungsgruppen in die Parteien. Weniger als ein Drittel der Personen mit niedriger und mittlerer Bildung vertraut den Parteien.

Differenziert nach Engagementstatus zeigt sich, dass Engagierte allen sechs hier aufgeführten Institutionen anteilig häufiger vertrauen als Nicht-Engagierte (Abbildung 14-5). Sowohl unter den Engagierten als auch unter den Nicht-Engagierten ist die Reihenfolge der Institutionen dieselbe: Der Polizei vertrauen 92,2 Prozent der Engagierten und 88,7 Prozent der Nicht-Engagierten. Unter den Engagierten vertrauen 83,7 Prozent der Justiz, unter den Nicht-Engagierten sind es lediglich 74,9 Prozent. Bei dieser Institution ist der Unterschied zwischen Engagierten und Nicht-Engagierten mit 8,8 Prozentpunkten am größten. Dem Bundestag vertrauen 62,5 Prozent der

Abbildung 14-5 Vertrauen in unterschiedliche Institutionen 2019, nach Engagementstatus (Basis: alle Befragten)

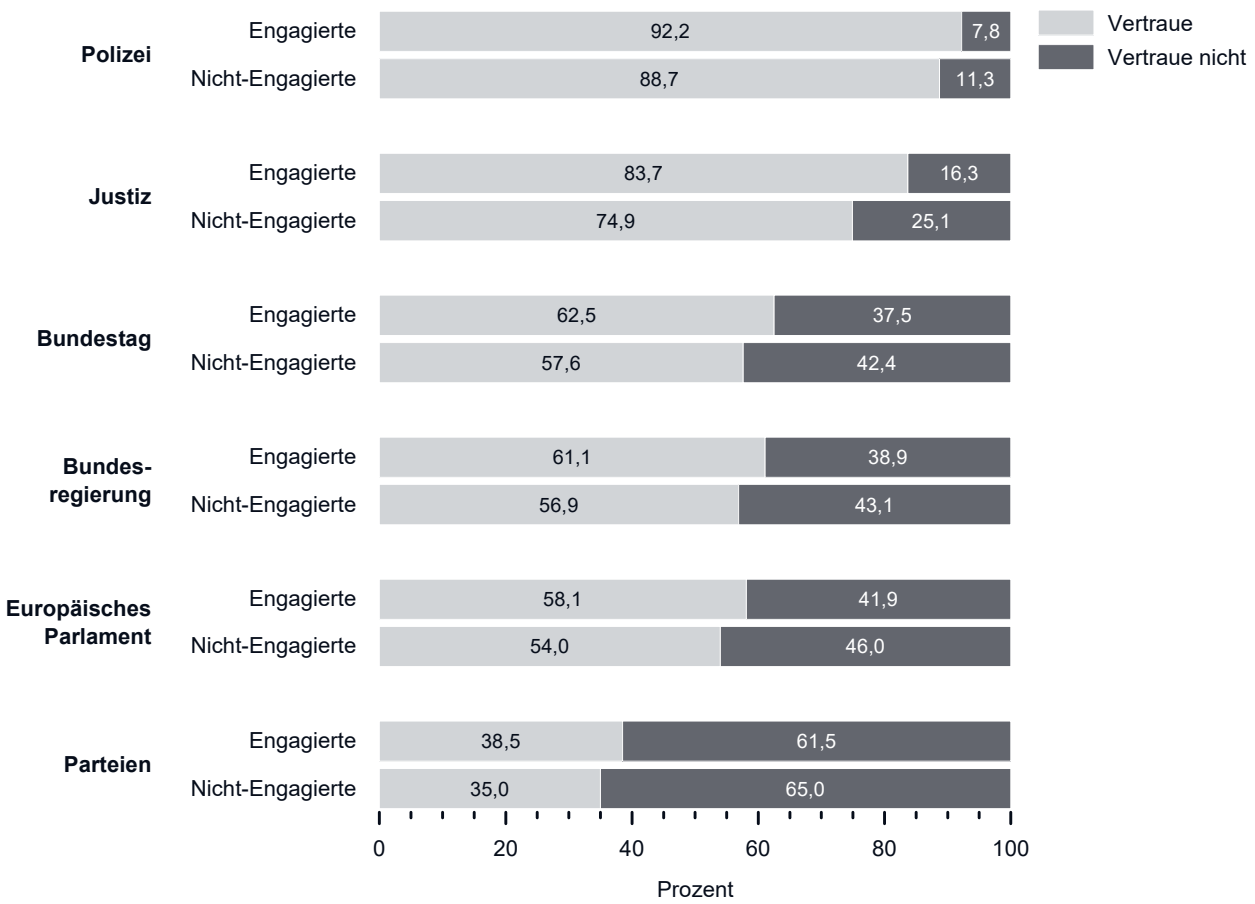

Quelle: FWS 2019, gewichtet, eigene Berechnungen (DZA). Basis: alle Befragten ( $n=26.401-27.411)$.

Alle Unterschiede zwischen Engagierten und Nicht-Engagierten sind statistisch signifikant. 
Engagierten und 57,6 Prozent der Nicht-Engagierten. Ein Anteil von 61,1 Prozent der Engagierten und 56,9 Prozent der Nicht-Engagierten vertraut der Bundesregierung. Dem Europäischen Parlament vertrauen 58,1 Prozent der Engagierten und 54,o Prozent der Nicht-Engagierten. Den Parteien wird mit 38,5 Prozent unter den Engagierten und 35,o Prozent unter den Nicht-Engagierten am seltensten vertraut.

\subsection{Einstellungen zur Demokratie als Regierungsform und zur Demokratie in Deutschland}

Eine große Mehrheit von 90,7 Prozent der Menschen ab 14 Jahren in Deutschland hält die Demokratie für eine gute Regierungsform; im Umkehrschluss heißt dies, dass knapp jede zehnte Person die Demokratie für eine nicht so gute Regierungsform hält (Abbildung 14-6a). Zwischen Frauen und Männern zeigen sich dabei nur geringe Unterschiede. Jüngere Personen zwischen 14 und 49 Jahren bewerten die Demokratie anteilig etwas häufiger als gute Regierungsform als Personen ab 50 Jahren.

Die größten Unterschiede zeigen sich zwischen den Bildungsgruppen (Abbildung 14-6b). Es ist ein deutlicher Bildungsgradient zu erkennen: 82,7 Prozent der Personen mit niedriger Bildung bewerten die Demokratie als gute Regierungsform. Unter den Personen mit mittlerer Bildung bewerten 89,9 Prozent die Demokratie als gute Regierungsform. Die höchsten Werte weisen die Personen mit hoher Bildung sowie die Personen, die noch eine Schule besuchen, auf.

Während die Demokratie als Regierungsform von neun von zehn Personen als gut bewertet wird, wird die praktische Umsetzung dieser in Deutschland deutlich kritischer gesehen. Zufrieden mit dem Funktionieren der Demokratie in Deutschland sind etwas mehr als zwei Drittel der Personen (68,4 Prozent), ein knappes Drittel (31,6 Prozent) ist mit dem Funktionieren der Demokratie in Deutschland nicht zufrieden (Abbildung 14-7a). Während sich zwischen den Geschlechtern keine Unterschiede hinsichtlich der Zufriedenheit zeigen, werden Unterschiede zwischen den Altersgruppen in diesem Zusammenhang deutlich. Anteilig am häufigsten sind die Personen zwischen 50 und 64 Jahren nicht zufrieden mit dem Funktionieren der Demokratie. Die Personen ab 65 Jahren geben anteilig etwas seltener an nicht zufrieden zu sein. Die Personen der beiden jüngsten Altersgruppen zwischen 14 und 49 Jahren geben anteilig am seltensten an nicht zufrieden $\mathrm{zu}$ sein und unterscheiden sich dahingehend nicht voneinander.

Die größten Unterschiede werden zwischen den jeweiligen Bildungsgruppen ersichtlich (Abbildung 14-7b). Personen mit niedriger Bildung sind anteilig am häufigsten nicht zufrieden mit dem Funktionieren der Demokratie in Deutschland (41,o Prozent), gefolgt von den Personen mit mittlerer Bildung (37,4 Prozent) und den Personen mit hoher Bildung (21,6 Prozent). Lediglich ein Anteil von 16,3 Prozent der Personen, die noch eine Schule besuchen, ist nicht zufrieden mit dem Funktionieren der Demokratie. 
Abbildung 14-6 Einstellungen zur Demokratie als Regierungsform 2019, a) gesamt, nach Geschlecht und nach Alter, b) nach Bildung (Basis: alle Befragten)

a) gesamt, nach Geschlecht und nach Alter

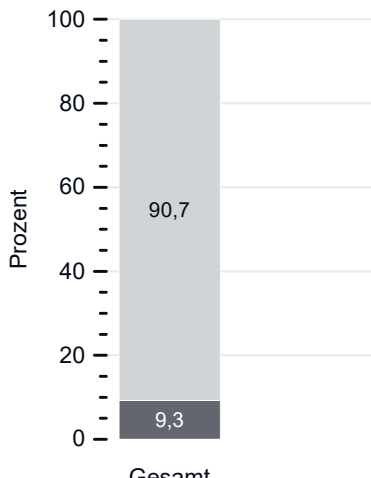

Gesamt

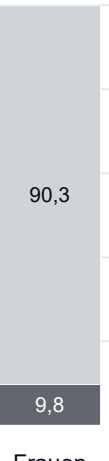

Frauen

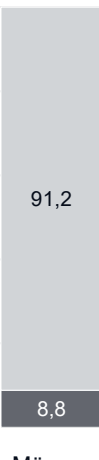

Männer

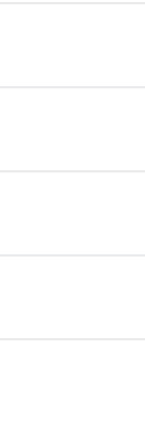

6,9

$14-29$

Jahre

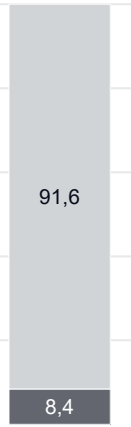

30-49

Jahre
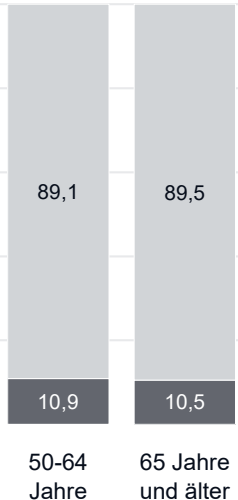

b) nach Bildung

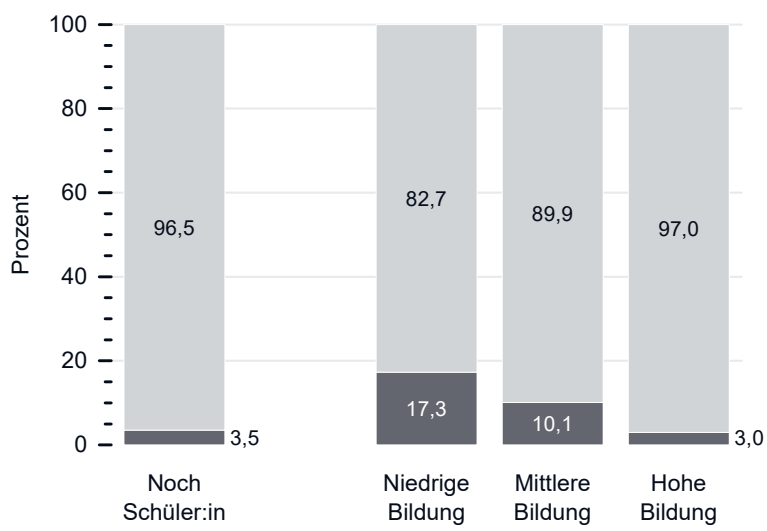

Gute Regierungsform

Nicht so gute Regierungsform

Quelle: FWS 2019, gewichtet, eigene Berechnungen (DZA). Basis: alle Befragten ( $n=27.317)$, nach Bildung $(n=27.312)$.

Hinweis: Abweichungen in den Prozentwerten ergeben sich durch das Auf- und Abrunden der Werte.

Der Geschlechterunterschied ist nicht statistisch signifikant.

Statistisch signifikanter Unterschied zwischen den 14- bis 29-Jährigen und den 50- und 64-Jährigen sowie den 65-Jährigen und Älteren. Statistisch signifikanter Unterschied zwischen den 30- bis 49-Jährigen und den 50- und 64-Jährigen sowie den 65-Jährigen und Älteren. Kein statistisch signifikanter Unterschied zwischen den 14- bis 29-Jährigen und den 30- bis 49-Jährigen sowie zwischen den 50- bis 64-Jährigen und den Personen ab 65 Jahren.

Statistisch signifikanter Unterschied zwischen Personen mit niedriger und mittlerer Bildung und jeweils allen anderen Bildungsgruppen. Kein statistisch signifikanter Unterschied zwischen Personen, die noch die Schule besuchen, und Personen mit hoher Bildung. 
Abbildung 14-7 Einstellungen zum Funktionieren der Demokratie in Deutschland 2019, a) gesamt, nach Geschlecht und nach Alter, b) nach Bildung (Basis: alle Befragten)

a) gesamt, nach Geschlecht und nach Alter

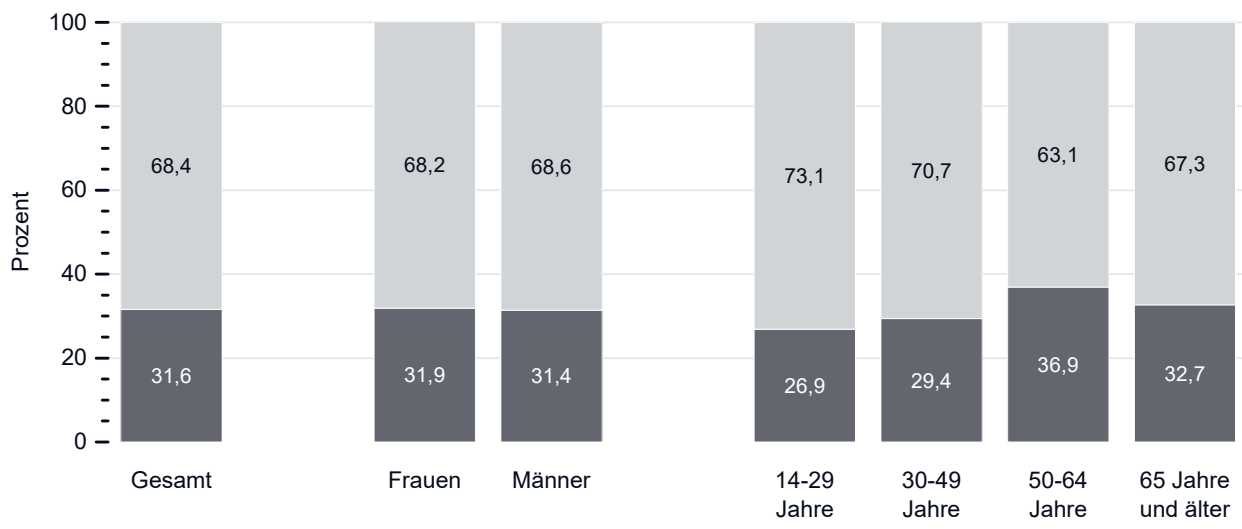

b) nach Bildung

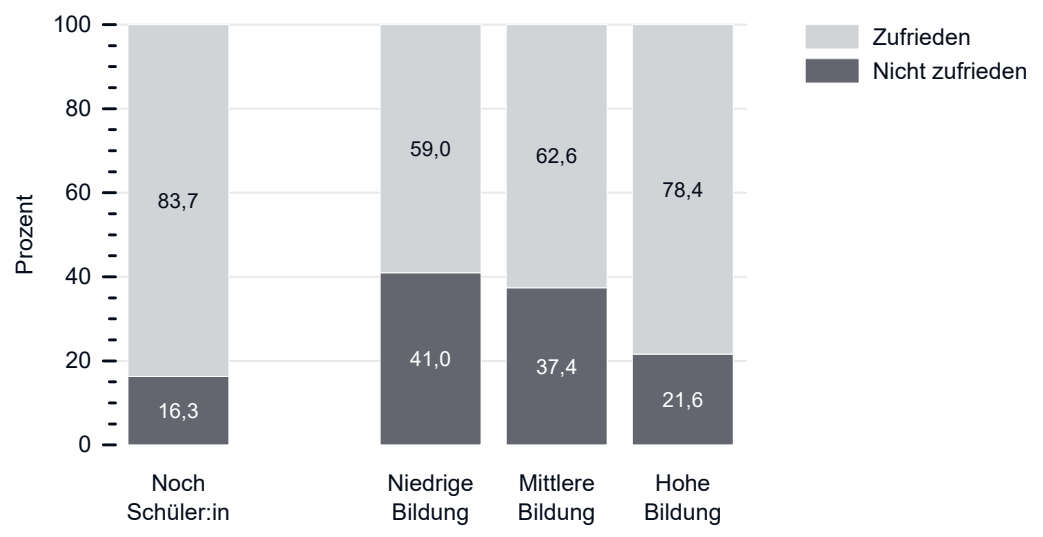

Quelle: FWS 2019, gewichtet, eigene Berechnungen (DZA). Basis: alle Befragten ( $n=27.459)$, nach Bildung $(n=27.454)$.

Hinweis: Abweichungen in den Prozentwerten ergeben sich durch das Auf- und Abrunden der Werte.

Der Geschlechterunterschied ist nicht statistisch signifikant.

Statistisch signifikanter Unterschied zwischen den 50- bis 64-Jährigen sowie den 65-Jährigen und Älteren und jeweils allen anderen Altersgruppen. Kein statistisch signifikanter Unterschied zwischen den 14- bis 29-Jährigen und den 30- bis 49-Jährigen.

Statistisch signifikanter Unterschied zwischen allen Bildungsgruppen. 
Zwischen der Einstellung zur Demokratie als Regierungsform und der Zufriedenheit mit dem Funktionieren der Demokratie in Deutschland zeigt sich eine deutliche Diskrepanz. Der Anteil der Personen, die angeben, die Demokratie als gute Regierungsform zu bewerten, ist deutlich größer als der Anteil der Personen, die mit dem Funktionieren der Demokratie in Deutschland zufrieden sind. Vor diesem Hintergrund werden in den folgenden Analysen die beiden Abfragen zu Demokratieeinstellungen miteinander kombiniert und die Häufigkeitsverteilung der daraus resultierenden vier Gruppen dargestellt, um ein differenziertes Bild der unterschiedlichen Einstellungen zu erhalten (Abbildung 14-8). Der Anteil der Personen, die die Demokratie für eine nicht so gute Regierungsform halten und auch mit dem Funktionieren der Demokratie nicht zufrieden sind, ist mit 7,0 Prozent relativ gering. Die Gruppe der Personen, die - trotz Ablehnung der Demokratie - mit ihrem aktuellen Funktionieren in Deutschland zufrieden sind, stellt mit 2,2 Prozent die kleinste Gruppe dar. Personen, die Zustimmung zu der Demokratie als Regierungsform äußern, mit dem aktuellen Funktionieren der Demokratie aber nicht zufrieden sind, machen mit einem Anteil von 24,4 Prozent, also einem knappen Viertel, die zweitgrößte Gruppe aus. Die größte Gruppe bilden Personen, die die Demokratie als gute Regierungsform bewerten und mit ihrem Funktionieren in Deutschland zufrieden sind. Diese Angabe machen gut zwei Drittel der Personen (66,4 Prozent).

Die Verteilung für Frauen und Männer ist der Verteilung in der Gesamtbevölkerung sehr ähnlich; es gibt keine statistisch signifikanten Geschlechterunterschiede (Abbildung 14-8).

Zwischen den Altersgruppen zeigen sich hingegen einige Unterschiede (Abbildung 14-9). Personen ab 50 Jahren geben anteilig häufiger an, die Demokratie als nicht so gute Regierungsform zu bewerten und zudem nicht zufrieden mit dem Funktionieren der Demokratie in Deutschland zu sein, als die jüngeren Personen zwischen 14 und 49 Jahren. Keine Unterschiede zwischen den Altersgruppen gibt es unter den Personen, die die Demokratie als nicht so gute Regierungsform bewerten, mit ihrem Funktionieren in Deutschland aber zufrieden sind. Diese Anteile sind mit jeweils unter drei Prozent in allen Altersgruppen sehr gering. Die Gruppe, die die Demokratie als gute Regierungsform bewertet, während sie mit dem Funktionieren in Deutschland nicht zufrieden ist, ist bei den Personen ab 50 Jahren anteilig größer als bei den 14- bis 29-Jährigen. Den größten Anteil mit 28,1 Prozent haben hier die Personen zwischen 50 und 64 Jahren. Ein deutlicher Altersunterschied findet sich bei der Gruppe der Personen, die die Demokratie als gute Regierungsform bewerten und mit dem Funktionieren der Demokratie in Deutschland zufrieden sind: Unter den 14- bis 29-Jährigen liegt der Anteil bei 71,7 Prozent, unter den 50- bis 64-Jährigen lediglich bei 61,o Prozent.

Nach Bildung differenziert sehen wir deutliche Unterschiede hinsichtlich der Einstellungen zur Demokratie (Abbildung 14-10). Die Personen mit niedriger Bildung geben anteilig häufiger als alle anderen Bildungsgruppen an, die Demokratie als nicht so gute Regierungsform zu bewerten und nicht zufrieden mit ihrem Funk- 


\section{Abbildung 14-8 Einstellungen zur Demokratie als Regierungsform und zum Funktionieren der Demokratie in Deutschland, 2019, gesamt und nach Geschlecht (Basis: alle Befragten)}

Gesamt

7,0

24,4

66,4

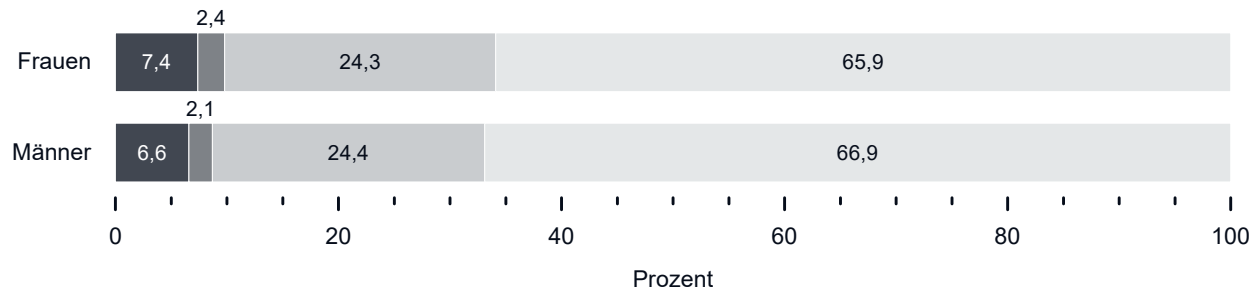

Prozent

$\begin{array}{llll}\text { Nicht so gute } & \text { Nicht so gute } & \text { Gute } & \text { Gute } \\ \begin{array}{l}\text { Regierungsform, } \\ \text { nicht zufrieden }\end{array} & \text { Regierungsform, } & \text { Regierungsform, } & \text { Regierungsform, } \\ \text { zufrieden } & \text { nicht zufrieden } & \text { zufrieden }\end{array}$

Quelle: FWS 2019, gewichtet, eigene Berechnungen (DZA). Basis: alle Befragten ( $n=27.123)$, nach Geschlecht $(n=27.123)$.

Die Geschlechterunterschiede sind statistisch nicht signifikant.

Abbildung 14-9 Einstellungen zur Demokratie als Regierungsform und zum Funktionieren der Demokratie in Deutschland, 2019, nach Alter (Basis: alle Befragten)

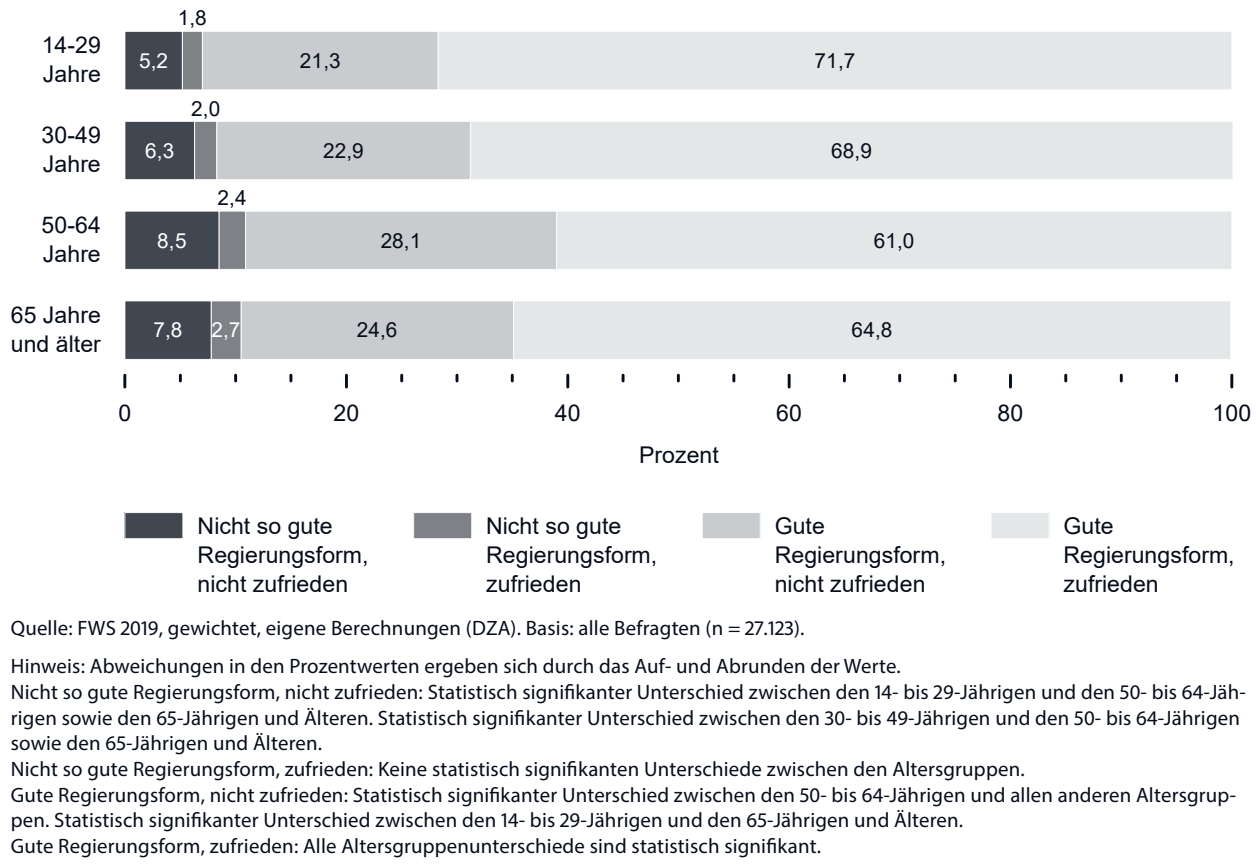


tionieren in Deutschland zu sein (13,7 Prozent). Unter den Befragten mit mittlerer Bildung ist dieser Anteil um 6,1 Prozentpunkte geringer. Personen, die aktuell die Schule besuchen, und Personen mit hoher Bildung weisen hier anteilig deutlich geringere Werte auf. Personen mit niedriger Bildung geben anteilig auch häufiger als alle anderen Bildungsgruppen an, die Demokratie als nicht so gute Regierungsform zu bewerten, mit dem Funktionieren dieser aber zufrieden zu sein. Auch unter den Personen, die die Demokratie als gute Regierungsform bewerten, mit ihrem Funktionieren in Deutschland aber unzufrieden sind, zeigen sich statistisch signifikante Unterschiede zwischen allen Bildungsgruppen. Diese Angaben machen Personen mit niedriger und mittlerer Bildung (26,8 Prozent sowie 29,9 Prozent) anteilig deutlich häufiger als Personen mit hoher Bildung sowie Personen, die noch zur Schule gehen (19,5 Prozent sowie 14,7 Prozent). Einen deutlichen Bildungsunterschied sehen wir bei den Personen, die die Demokratie als gute Regierungsform bewerten und mit dem Funktionieren der Demokratie in Deutschland zufrieden sind: Lediglich ein Anteil von 55,9 Prozent der Menschen mit niedriger Bildung gehört zu dieser Gruppe, während es bei Menschen mit hoher Bildung 77,5 Prozent und bei Personen, die noch die Schule besuchen, sogar 81,8 Prozent sind.

Abbildung 14-10 Einstellungen zur Demokratie als Regierungsform und zum Funktionieren der Demokratie in Deutschland, 2019, nach Bildung (Basis: alle Befragten)

\begin{tabular}{c|cc} 
Noch & 1,9 & \\
Schüler:in & 14,6 & 81,8
\end{tabular}

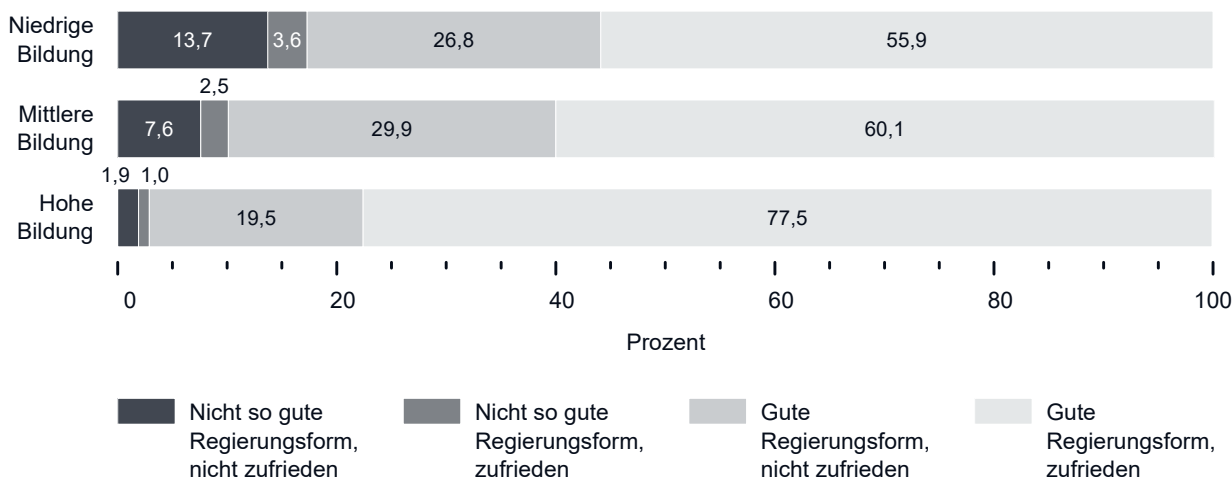

Quelle: FWS 2019, gewichtet, eigene Berechnungen (DZA). Basis: alle Befragten ( $n=27.118)$.

Hinweis: Abweichungen in den Prozentwerten ergeben sich durch das Auf- und Abrunden der Werte.

Nicht so gute Regierungsform, nicht zufrieden: Statistisch signifikanter Unterschied zwischen den Personen mit niedriger Bildung und allen weiteren Bildungsgruppen. Statistisch signifikanter Unterschied zwischen den Personen mit mittlerer Bildung und allen weiteren Bildungsgruppen.

Nicht so gute Regierungsform, zufrieden: Statistisch signifikanter Unterschied zwischen den Personen mit niedriger Bildung und allen anderen Bildungsgruppen sowie zwischen Personen mit mittlerer Bildung und hoher Bildung.

Gute Regierungsform, nicht zufrieden: Statistisch signifikanter Unterschied zwischen allen Bildungsgruppen.

Gute Regierungsform, zufrieden: Statistisch signifikanter Unterschied zwischen allen Bildungsgruppen. 


\subsection{Einstellungen zur Demokratie im Kontext des freiwilligen Engagements}

Zwischen Engagierten und Nicht-Engagierten zeigen sich statistisch signifikante Unterschiede hinsichtlich der Einstellungen gegenüber der Demokratie. Ein Anteil von 95, o Prozent der Engagierten bewertet die Demokratie als gute Regierungsform (Abbildung 14-11a). Unter den Nicht-Engagierten bewerten 87,8 Prozent die Demokratie als gute Regierungsform.

Die Zufriedenheit mit dem Funktionieren der Demokratie ist sowohl unter den Nicht-Engagierten als auch unter den Engagierten deutlich geringer (Abbildung 1411b). Ein Anteil von 72,2 Prozent der Engagierten ist mit dem Funktionieren der Demokratie zufrieden. Nicht-Engagierte sind mit 65,8 Prozent anteilig seltener zufrieden.

Abbildung 14-11 Einstellungen a) zur Demokratie als Regierungsform, 2019, nach Engagementstatus und b) zum Funktionieren der Demokratie in Deutschland, 2019, nach Engagementstatus (Basis: alle Befragten)

a) Einstellung zur Demokratie als Regierungsform

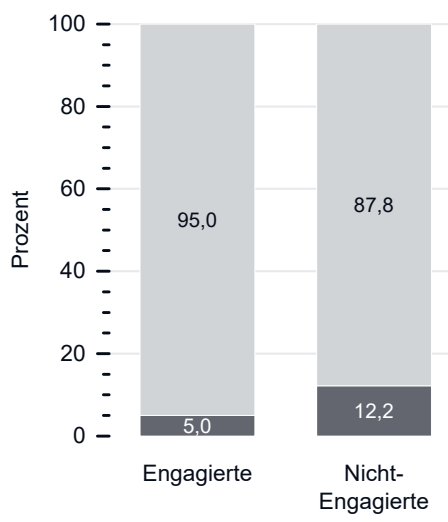

Gute Regierungsform

Nicht so gute Regierungsform b) Einstellung zum Funktionieren der Demokratie in Deutschland

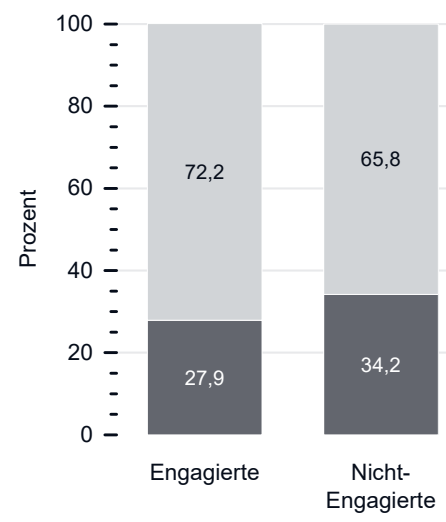

Zufrieden

Nicht zufrieden

Quelle: FWS 2019, gewichtet, eigene Berechnungen (DZA). Basis: alle Befragten. Demokratie als Regierungsform $(n=27.314)$, Demokratie in Deutschland $(n=27.456)$.

Hinweis: Abweichungen in den Prozentwerten ergeben sich durch das Auf- und Abrunden der Werte.

Der Unterschied zwischen Engagierten und Nicht-Engagierten ist für beide Indikatoren statistisch signifikant. 
Der Anteil der Personen, die die Demokratie als nicht so gute Regierungsform bewerten und mit dem Funktionieren der Demokratie nicht zufrieden sind, ist mit 9,4 Prozent unter den Nicht-Engagierten deutlich größer als unter den Engagierten mit 3,6 Prozent (Abbildung 14-12). Auch der Anteil der Personen, die trotz einer Bewertung der Demokratie als nicht so gute Regierungsform mit dem Funktionieren der Demokratie in Deutschland zufrieden sind, liegt bei den Nicht-Engagierten mit 2,8 Prozent über dem Anteil von 1,4 Prozent bei Engagierten. Bei der Bewertung der Demokratie als gute Regierungsform unterscheiden sich die Engagierten und die Nicht-Engagierten, die unzufrieden mit dem Funktionieren der Demokratie in Deutschland sind, nicht voneinander. Ein deutlicher Unterschied zeigt sich jedoch bei denjenigen, die die Demokratie als gute Regierungsform bewerten und mit dem Funktionieren der Demokratie zufrieden sind: Diese Angaben machen 71,o Prozent der Engagierten und 63,3 Prozent der Nicht-Engagierten.

Abbildung 14-12 Einstellungen zur Demokratie als Regierungsform und zum Funktionieren der Demokratie in Deutschland, 2019, nach Engagementstatus (Basis: alle Befragten)

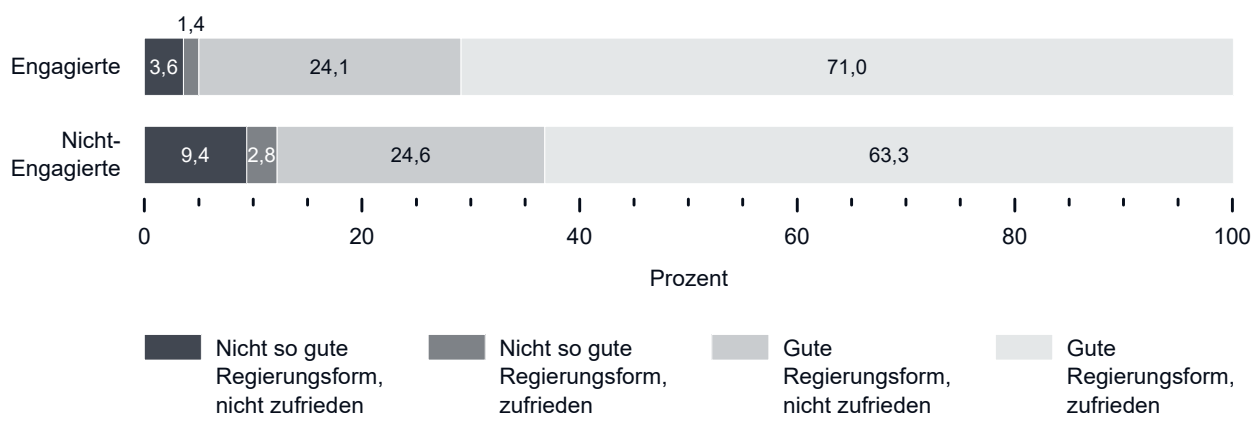

Quelle: FWS 2019, gewichtet, eigene Berechnungen (DZA). Basis: alle Befragten $(n=27.120)$.

Hinweis: Abweichungen in den Prozentwerten ergeben sich durch das Auf- und Abrunden der Werte.

Statistisch signifikanter Unterschied zwischen den Engagierten und den Nicht-Engagierten in allen Kategorien außer,Gute Regierungsform, nicht zufrieden'.

\subsection{Fazit}

Das Vertrauen in grundlegende gesellschaftliche und demokratische Institutionen und die Akzeptanz der Demokratie sind wichtige Voraussetzungen für eine funktionierende demokratische Gesellschaft. Die Ergebnisse dieses Kapitels zeigen, dass die große Mehrheit der Bevölkerung in Deutschland der Demokratie als Regierungsform positiv gegenübersteht. Diese Befunde decken sich weitgehend mit den Ergebnissen anderer Studien (zum Beispiel Bertelsmann Stiftung 2019; Decker et al. 2018; Decker et al. 2019; Stroppe \& Mauk 2021; Jacobsen \& Kroh 2018). 
Allerdings ist etwa ein Drittel der Bevölkerung mit dem Funktionieren der Demokratie in Deutschland nicht zufrieden. Dieses Unbehagen an der realen Umsetzung der Demokratie kann verschiedene Ursachen haben. Möglicherweise liegt einer der Gründe darin, dass offene, plurale und konfliktreiche politische Prozesse, die zum Wesen der Demokratie gehören, weniger akzeptiert werden als die Demokratie als Ideal (König 2017). Ein anderer Grund für die Unzufriedenheit mit der Demokratie wird unter dem Stichwort des ,Responsivitätsmangels' diskutiert: Die Wahrnehmung, dass sich politische Akteure nicht ausreichend um die Belange der Bevölkerung kümmern, kann zu Unzufriedenheit mit der Demokratie führen (Busch 2020). Schließlich werden als Faktoren, die auf die Zufriedenheit der Menschen mit dem Funktionieren der Demokratie wirken, die individuelle wirtschaftliche Lage sowie die wirtschaftliche Lage des Landes genannt (Busch 2020). Demokratie wird mit einem Wohlstandsversprechen verbunden (Münkler 2020), welches mancherorts - besonders in strukturschwachen Regionen - vermutlich nicht erfüllt werden kann. In diesem Zusammenhang wird berichtet, dass insbesondere die europäischen Länder, die stark unter globalen Krisen leiden, einen Abfall der Demokratiezufriedenheit zu verzeichnen haben. Ein Beispiel dafür ist Griechenland während der weltweiten Wirtschaftskrise ab 2007 (Fuchs \& Roller 2019). Liegt die Zufriedenheit mit dem Funktionieren der Demokratie bei über 6o Prozent - wie dies in den Befunden des Freiwilligensurveys 2019 der Fall ist -, so wird nicht von einer Krise des Vertrauens in die Demokratie ausgegangen (Fuchs \& Roller 2019). Es wird jedoch angemerkt, dass eine andauernde Unzufriedenheit mit dem Funktionieren der Demokratie sich langfristig negativ auf die Präferenz der Demokratie als Regierungsform auswirken kann (Busch 2020; Fuchs \& Roller 2019). Angesichts des hohen Zuspruchs für die Demokratie als Regierungsform von über 90 Prozent, kann aber aktuell nicht von einer Legitimitätskrise der Demokratie ausgegangen werden (Fuchs \& Roller 2019).

Ein vergleichsweiser geringer Teil von insgesamt 2,2 Prozent der Personen gibt an, die Demokratie abzulehnen, mit dem Funktionieren der Demokratie in Deutschland jedoch zufrieden zu sein. Diese Gruppe kann die Demokratie grundsätzlich aus ideologischen Gründen ablehnen, beziehungsweise andere Herrschaftsformen bevorzugen, und unabhängig davon mit dem Funktionieren der demokratischen Strukturen - insbesondere in regionalen Kontexten - und deren Einfluss auf die eigene Lebenssituation zufrieden sein. Die Ablehnung des Systems ist in diesem Fall unabhängig von den Leistungen des Systems (Bertelsmann Stiftung 2019). Personen mit niedriger Bildung sowie Nicht-Engagierte sind in dieser Gruppe anteilig häufiger zu finden als die Personen der jeweiligen Vergleichsgruppen.

Das Vertrauen in wichtige Institutionen der Demokratie ist für diejenigen Institutionen, die Sicherheit (Polizei) und Recht (Justiz) garantieren, am höchsten. Deutlich niedriger ist das Vertrauen in die Institutionen der parlamentarischen Demokratie Bundestag und Europäisches Parlament sowie Bundesregierung. Parteien, denen es als politischen Akteuren zukommt, unterschiedliche gesellschaftliche Positionen im parlamentarischen Prozess auszuhandeln, wird das geringste Vertrauen entgegen- 
gebracht. Ähnliche Muster des Institutionenvertrauens finden sich auch in anderen Studien (Bertelsmann Stiftung 2019; Reuband 2012). Einerseits wird das niedrige Vertrauen der Bevölkerung in Parteien als kritische Betrachtung gewertet, andererseits wird es aber auch als für das Funktionieren demokratischer Prozesse bedenklich betrachtet, da den Parteien die Aufgabe der Willensbündelung und -artikulation in der Demokratie zukommt. Wenn Personen, die den Parteien misstrauen, sich auf Grund dessen von den Parteien abwenden, so werden ihre Belange weniger gehört und vertreten. Es besteht darüber hinaus die Gefahr, dass sich das Misstrauen in einer Unzufriedenheit und auch einer Ablehnung demokratischer Strukturen manifestiert. Es ist auch aus anderen Studien bekannt, dass jene Institutionen, die wenig oder gar nicht in politische Entscheidungsfindungen eingebunden sind und daher als unparteiisch oder neutral gelten, ein höheres Vertrauen in der Bevölkerung genießen (zum Beispiel Bertelsmann Stiftung 2019).

Unterschiede zwischen Frauen und Männern bei der Beurteilung der Demokratie als Regierungsform, der Zufriedenheit mit dem Funktionieren der Demokratie in Deutschland sowie beim Vertrauen in gesellschaftliche Institutionen sind nur gering. Anders sieht es mit Blick auf Alters- und Bildungsgruppen aus, unter denen erhebliche Unterschiede hinsichtlich der Einstellungen zur Demokratie und dem Institutionenvertrauen bestehen. Die Einstellungen zur Demokratie und das Institutionenvertrauen fallen bei jüngeren Menschen insgesamt positiver aus als bei älteren Menschen. Auffällig dabei ist, dass die geringsten Zustimmungsquoten zur Demokratie und das geringste Institutionenvertrauen bei den 50- bis 64-Jährigen zu finden sind. Bei den 65-Jährigen und Älteren sind die Zustimmungsquoten und das Institutionenvertrauen etwas höher, liegen aber noch deutlich unter den Werten der jüngsten Altersgruppe. Ob sich diese Unterschiede auf das Lebensalter zurückführen lassen oder ob hier Effekte eine Rolle spielen, die mit den unterschiedlichen Geburtenjahrgängen zusammenhängen (Kohorteneffekte), kann anhand der vorliegenden Daten nicht geklärt werden.

Die Unterschiede zwischen Bildungsgruppen in der Einstellung zur Demokratie und im Institutionenvertrauen sind groß. Menschen mit niedriger Bildung stehen der Demokratie ablehnender und kritischer gegenüber als Menschen mit hoher Bildung, und auch ihr Institutionenvertrauen ist deutlich geringer ausgeprägt. Der Anteil der Personen, die die Demokratie als gute Regierungsform bewerten und mit dem Funktionieren zufrieden sind, ist in der Gruppe der Personen mit niedriger Bildung besonders niedrig, bei Menschen mit hoher Bildung deutlich höher. Ähnliche Befunde gibt es auch in anderen Studien (zum Beispiel Lange 2018). Zudem sind Personen mit niedriger Bildung anteilig häufiger nicht zufrieden mit dem Funktionieren der Demokratie in Deutschland als Personen mit hoher Bildung. Dies könnte mit einem Mangel an Repräsentativität zusammenhängen. So sind in den Parlamenten vorrangig Menschen mit hoher Bildung zu finden und Institutionen werden von Personen mit hoher Bildung repräsentiert (Bovens \& Wille 2017). Wenn sich die Personen mit niedriger Bildung demnach nicht vertreten sehen, kann dies zu einer Unzufrieden- 
heit mit dem Funktionieren der Demokratie führen (Busch 2020). Weiterhin spielt auch die häufig unterschiedliche sozioökonomische Basis der unterschiedlichen Bildungsgruppen eine Rolle: Personen mit hoher Bildung verfügen meist über höhere finanzielle Ressourcen und ein höheres Einkommen. Personen mir niedriger Bildung sind häufiger mit geringeren sozioökonomischen Ressourcen ausgestattet und somit eher von Statusunsicherheit betroffen (Lengfeld 2018). Die Ablehnung oder Skepsis gegenüber der Demokratie kann aus dieser relativen Unsicherheit resultieren. Diese Annahme deckt sich mit dem Befund, dass die individuelle wirtschaftliche Lage Einfluss auf die Zufriedenheit mit der Demokratie wie auch langfristig auf die Bewertung der Demokratie als Herrschaftsform hat (Busch 2020). Hier herrscht Handlungsbedarf für die Politik, eine gute ökonomische Basis sowie Bildung für alle Menschen in Deutschland zu schaffen und den Belangen der Menschen nachzukommen.

Freiwilliges Engagement geht mit einer positiveren Einstellung zur Demokratie, einer höheren Zufriedenheit mit dem Funktionieren der Demokratie sowie einem stärkeren Vertrauen in gesellschaftliche Institutionen einher. Die Unterschiede zwischen freiwillig Engagierten und Nicht-Engagierten sind dabei nicht durchgängig sehr groß, aber in allen Indikatoren deutlich sichtbar. Hier könnten sozioökonomische Faktoren eine wichtige Rolle spielen. Es ist bekannt, dass sich Personen mit einer geringeren Bildung sowie einem geringeren Einkommen anteilig seltener freiwillig engagieren als Personen mit einer hohen sozioökonomischen Ressourcenausstattung (siehe Kapitel 4). Gleichzeitig können Personen mit einer geringeren Ressourcenausstattung auch die Zugangschancen für freiwilliges Engagement fehlen (Simonson \& Hameister 2017). Hinter der anteilig höheren positiven Einstellung gegenüber der Demokratie unter den Engagierten kann sich somit auch eine höhere Ressourcenausstattung verbergen, die sowohl zu einer positiveren Demokratieeinstellung als auch zu einer höheren Engagementwahrscheinlichkeit führt.

Es könnte aber auch sein, dass diese Befunde einen Wirkungszusammenhang zwischen Zivilgesellschaft, Engagement und Demokratie zeigen (Zimmer 2010). Dabei könnte es sich um eine wechselseitige Befruchtung von freiwilligem Engagement und einer positiven Einstellung zur Demokratie handeln. Befunde über einen direkten Zusammenhang zwischen freiwilligem Engagement und dem Wunsch nach aktiver Beteiligung bei der Gestaltung der Zivilgesellschaft finden sich auch bei den Motiven für die Ausübung freiwilligen Engagements wieder: Das Motiv, die Gesellschaft mitgestalten zu wollen, geben vier von fünf Engagierte an (siehe Kapitel 6). Es ist jedoch genau zu betrachten, ob die Beteiligung an der Zivilgesellschaft möglicherweise auf die Schwächung oder gar Abschaffung der Demokratie abzielt. Jede zwanzigste engagierte Person bewertet die Demokratie als nicht so gute Regierungsform und könnte so potenziell demokratiefeindliche Einstellungen in das freiwillige Engagement einbringen. Die Befunde dieses Kapitels verdeutlichen, dass es wichtig ist, auf entsprechende Strukturen innerhalb des Engagements zu reagieren und weitere Präventionsarbeit zu leisten. Insgesamt sind die Anteile der Personen, die potenziell demokratiefeindlich eingestellt sind, unter allen Befragten und insbesondere unter den 
Engagierten eher gering. Auch wenn die vorliegenden Daten es nicht erlauben, Kausalschlüsse zu ziehen, wird anhand dieser Befunde deutlich, dass sich einerseits Menschen mit positiver Einstellung zur Demokratie und hohem Institutionenvertrauen am freiwilligen Engagement beteiligen, diese Beteiligung in der Zivilgesellschaft aber auch zu einer Stärkung des Vertrauens in Institutionen und einer positiven Einstellung zur Demokratie insgesamt und zum Funktionieren der Demokratie in Deutschland führen könnte.

\section{Literatur}

Alscher, M., Priller, E., \& Burkhardt, L. (2021). Zivilgesellschaftliches Engagement. In: Statistisches Bundesamt (Destatis), Wissenschaftszentrum Berlin für Sozialforschung (WZB) \& Bundesinstitut für Bevölkerungsforschung (BiB) (Hrsg.) Datenreport 2021. Ein Sozialbericht für die Bundesrepublik Deutschland (S. 399-407). Bonn: Bundeszentrale für politische Bildung.

Bertelsmann Stiftung (2019). Schwindendes Vertrauen in Politik und Parteien. Eine Gefahr für den gesellschaftlichen Zusammenhalt? Gütersloh: Bertelsmann Stiftung.

Bovens, M., \& Wille, A. (2017). A not so universal suffrage: How Europe's political elites have become educational elites. Blog-Beitrag auf EUROPP - European Politics and Policy der London School of Economics and Political Science. Online: https://blogs.lse.ac.uk/ europpblog/2017/10/24/a-not-so-universal-suffrage-how-europes-political-elites-havebecome-educational-elites/ (zuletzt abgerufen 30.10.2020).

Bundesregierung (2016). Strategie der Bundesregierung zur Extremismusprävention und Demokratieförderung. Berlin: Bundesministerium für Familie, Senioren, Frauen und Jugend (BMFSFJ).

Busch, K. (2020). Krise oder Unterstützung der (repräsentativen) Demokratie in Deutschland? Die Entwicklung der letzten zwei Jahrzehnte 1991-2018. Informationsdienst Soziale Indikatoren, 65, 7-14.

Decker, F., Best, V., Fischer, S., \& Küppers, A. (2019). Vertrauen in Demokratie. Wie zufrieden sind die Menschen in Deutschland mit Regierung, Staat und Politik? Berlin: Friedrich-EbertStiftung.

Decker, O., Schuler, J., \& Brähler, E. (2018). Das autoritäre Syndrom heute. In: O. Decker \& E. Brähler in Zusammenarbeit mit der Heinrich-Böll-Stiftung und der Otto Brenner Stiftung (Hrsg.) Flucht ins Autoritäre. Rechtsextreme Dynamiken in der Mitte der Gesellschaft (S. 117156). Gießen: Psychosozial-Verlag.

Dietrich, K. (2019). Erzählungsbezogene Ansätze der Jugendarbeit. Zur Arbeit an lebensweltbasierten Ablehnungshaltungen. In: L. Boehnke, M. Thran \& J. Wunderwald (Hrsg.) Rechtspopulismus im Fokus. Theoretische und praktische Herausforderungen für die politische Bildung (S. 233-252). Wiesbaden: Springer VS.

Fuchs, D. \& Roller, E. (2019). Die Legitimitätsproblematik in den westeuropäischen Demokratien. In: C. Wiesner \& P. Harfst (Hrsg.) Legitimität und Legitimation - Vergleichende Perspektiven (S. 139-170). Wiesbaden: Springer VS. 
Geißel, B. (2006). Kritische Bürgerinnen und Bürger - eine Gefahr für Demokratien? Aus Politik und Zeitgeschichte (APuZ), (12), 3-9.

Jacobsen, J., \& Kroh, M. (2018). Einstellungen zur Demokratie in Deutschland und im internationalen Vergleich. GWP - Gesellschaft. Wirtschaft. Politik, 67(2), 233-241.

Jesse, E. (2020). Die Gefährdung der Demokratie in Deutschland: Krise oder Alarmismus? Zeitschrift für Gemeinschaftskunde, Geschichte und Wirtschaft, 79(37), 36-45.

Kalb, J. (2020). Die Pandemie "Covid-19“ (das Corona-Virus) als Herausforderung für freiheitliche Gesellschaften. Zeitschrift für Gemeinschaftskunde, Geschichte und Wirtschaft, 79(37), 3-7.

König, P.D. (2017). Von Demokraten und Populisten - Eine Analyse der Verbreitung unterschiedlicher Erwartungen an die Demokratie in Deutschland. Zeitschrift für Politikwissenschaft, 27(1), 1-28.

Küpper, B. \& Zick, A. (2020). Verlorene Mitte? (Anti-)demokratische und rechtsextreme Einstellungen in Deutschland. Zeitschrift für Gemeinschaftskunde, Geschichte und Wirtschaft, 79(37), $78-87$.

Lange, H. (2018). Determinanten der Demokratiezufriedenheit. Einfluss ökonomischer Faktoren auf die politische Kultur in der BRD. Wiesbaden: Springer.

Lengfeld, H. (2018). Der „Kleine Mann“ und die AfD: Was steckt dahinter? Kölner Zeitschrift für Soziologie und Sozialpsychologie, 7o(2), 295-310.

Münkler, H. (2020). Westliche Demokratien zwischen Partizipation und Wohlstand. Zeitschrift für Gemeinschaftskunde, Geschichte und Wirtschaft, 79(37), 16-25.

Pfahl-Traughber, A. (2019). Rechtsextremismus in Deutschland: Eine kritische Bestandsaufnahme. Wiesbaden: Springer VS.

Putnam, R. D. (2000). Bowling alone. The collapse and revival of American community. New York: Simon \& Schuster.

Reuband, K.-H. (2012). Vertrauen in die Polizei und staatliche Institutionen: Konstanz und Wandel in den Einstellungen der Bevölkerung 1984-2011. Soziale Probleme, 23(1), 5-39.

Simonson, J., \& Hameister, N. (2017). Sozioökonomischer Status und freiwilliges Engagement. In: J. Simonson, C. Vogel \& C. Tesch-Römer (Hrsg.) Freiwilliges Engagement in Deutschland Der Deutsche Freiwilligensurvey 2014 (S. 439-464). Wiesbaden: Springer VS.

Simonson, J., \& Vogel, C. (2017). Politische Partizipation: Unterschriftenaktionen, Demonstrationen, Bürgerinitiativen und politische Ämter. In: J. Simonson, C. Vogel \& C. Tesch-Römer (Hrsg.) Freiwilliges Engagement in Deutschland - Der Deutsche Freiwilligensurvey 2014 (S. 199216). Wiesbaden: Springer VS.

Simonson, J., Vogel, C., Ziegelmann, J.P., \& Tesch-Römer, C. (2017). Einleitung: Freiwilliges Engagement in Deutschland. In: J. Simonson, C. Vogel \& C. Tesch-Römer (Hrsg.) Freiwilliges Engagement in Deutschland - Der Deutsche Freiwilligensurvey 2014 (S. 31-49). Wiesbaden: Springer VS.

Spickschen, L., Meyer, D. \& Hilpert, W. (2020). Das Coronavirus und die Grundrechte. Bonn: Bundeszentrale für politische Bildung. Online: https://www.bpb.de/politik/grundfragen/politik-einfach-fuer-alle/309631/das-coronavirus-und-die-grundrechte (zuletzt abgerufen: 14. 09 . 2020). 
Stroppe, A.-K., \& Mauk, M. (2021). Einstellungen zu Demokratie und Sozialstaat. In: Statistisches Bundesamt (Destatis), Wissenschaftszentrum Berlin für Sozialforschung (WZB) \& Bundesinstitut für Bevölkerungsforschung (BiB) (Hrsg.) Datenreport 2021. Ein Sozialbericht für die Bundesrepublik Deutschland (S. 386-393). Bonn: Bundeszentrale für politische Bildung.

van Deth J. (2013) Sind Partizipierende die besseren Demokraten? In: S. Keil \& S. Thaidigsmann (Hrsg.) Zivile Bürgergesellschaft und Demokratie (S. 35-52). Wiesbaden: Springer VS.

Vorländer, H., Herold, M., \& Schäller, S. (2017): Entfremdung, Empörung, Ethnozentrismus. Was PEGIDA über den sich formierenden Rechtspopulismus verrät. In: D. Jörke, O. Nachtwey (Hrsg.) Das Volk gegen die (liberale) Demokratie; Sonderband Leviathan, Bd. 32 (S. 138-160). Baden-Baden: Nomos.

Zick, A., Krause, D., \& Küpper B. (2016). Rechtspopulistische und rechtsextreme Einstellungen in Deutschland. In: A. Zick, B. Küpper \& D. Krause (Hrsg.) Gespaltene Mitte - Feindselige Zustände (S. 111-142). Bonn: J. H. W. Dietz Nachf. GmbH.

Zick, A., \& Küpper B. (2016). Einleitung: Gespaltene Mitte, zerrissene Gesellschaft. In: A. Zick, B. Küpper \& D. Krause (Hrsg.) Gespaltene Mitte - Feindselige Zustände (S. 13-21). Bonn: J.H.W. Dietz Nachf. GmbH.

Zimmer, A. (2010). Zivilgesellschaft und Demokratie in Zeiten des gesellschaftlichen Wandels. dms - der moderne staat - Zeitschrift für Public Policy, Recht und Management, 3(1), 147-163.

Open Access Dieses Kapitel wird unter der Creative Commons Namensnennung Nicht kommerziell 4.0 International Lizenz (http://creativecommons.org/licenses/bync/4.0/deed.de) veröffentlicht, welche die nicht-kommerzielle Nutzung, Vervielfältigung, Bearbeitung, Verbreitung und Wiedergabe in jeglichem Medium und Format erlaubt, sofern Sie den/die ursprünglichen Autor(en) und die Quelle ordnungsgemäß nennen, einen Link zur Creative Commons Lizenz beifügen und angeben, ob Änderungen vorgenommen wurden.

Die in diesem Kapitel enthaltenen Bilder und sonstiges Drittmaterial unterliegen ebenfalls der genannten Creative Commons Lizenz, sofern sich aus der Abbildungslegende nichts anderes ergibt. Sofern das betreffende Material nicht unter der genannten Creative Commons Lizenz steht und die betreffende Handlung nicht nach gesetzlichen Vorschriften erlaubt ist, ist auch für die oben aufgeführten nicht-kommerziellen Weiterverwendungen des Materials die Einwilligung des jeweiligen Rechteinhabers einzuholen. 